\title{
Bifunctional nano-sponges serving as non-precious metal catalysts and self-standing cathodes for high performance fuel cell applications
}

Gang Yang ${ }^{+}$, Celal Erbay ${ }^{+}$, Su-in Yi, Paul de Figueiredo, Reza Sadr, Arum Han,* and Choongho $Y u^{*}$

G. Yang, Su-in Yi, Prof. C. Yu

Department of Mechanical Engineering

Texas A\&M University, College Station, Texas 77843, USA

E-mail: chyu@tamu.edu

C. Erbay, Prof. A. Han

Department of Electrical and Computer Engineering

Texas A\&M University, College Station, Texas 77843, USA

E-mail: arum.han@ece.tamu.edu

Prof. P. de Figueiredo

Department of Molecular Pathogenesis and Immunology

Texas A\&M Health Science Center, Bryan, Texas 77807, USA

Department of Veterinary Pathobiology

Texas A\&M University, College Station, Texas 77843, USA

Norman Borlaug Center

Texas A\&M University, College Station, Texas 77843, USA

Prof. R. Sadr

Mechanical Engineering Program

Texas A\&M University at Qatar, Doha, Qatar

${ }^{+}$These authors contributed equally to this work.

* Corresponding authors.

Keywords: microbial fuel cell, carbon nanotube sponge, long term stability, three-dimensional cathode, non-precious metal catalyst 


\begin{abstract}
In electrochemical cells, oxygen has been generally regarded as the ideal cathode reactant due to its non-toxicity, sustainability, and low-cost. However, the intrinsically slow oxygen reduction reaction (ORR) calls for electrocatalysts such as Pt and its alloys, and their high prices hamper the wide deployment of various electrochemical systems relying on ORR. Previously reported non-precious metal catalysts often involve complicated and lengthy synthesis processes as well as require additional catalyst loading electrodes, increasing the production complexity and cost of cathodes. Here we developed a bifunctional non-precious metal based electrocatalyst, which can also act as a self-standing sponge-like cathode, eliminating the usage of a catalyst loading/supporting layer. Our 3-dimensional (3D) catalysts/cathodes were tested in microbial fuel cells, showing outstanding catalytic activity and long term stability comparable to commercial Pt-based catalysts. Our cathodes were composed of self-assembled carbon nanotubes whose carbon is coordinated with iron and nitrogen for high ORR performance. For maximum cell performance, we found that the pore volume in the $3 \mathrm{D}$ cathode needs to be larger to have better oxygen diffusion but overly porous cathodes have less effective electrical conductivity, resulting in lower power generation. Our findings regarding the dependency of power generation on oxygen diffusion/reaction, effective electrical conductivity, and active surface area (or mass) provide a guidance to the future development of porous 3D electrocatalysts/cathodes. The new way of designing electrocatalysts/cathodes from conventional two-dimensional films to macroscale 3D self-assembled nanomaterials, with only $\sim 1 \%$ cost of commcial Pt-based catalyst powders, will elimnate one of major hurdles in deploying electrochemial energy conversion systems.
\end{abstract}


Microbial fuel cell (MFC) is a promising renewable energy technology for the production of electrical energy during wastewater treatment. ${ }^{[1]}$ Electrochemically active bacteria (EAB) oxidize organic compounds and transfer electrons to the anode. The electrons move through an external circuit to the cathode where the electron acceptors are reduced. ${ }^{[2]}$ Various electron acceptors such as ferricyanide and permanganate have been used in the cathode to improve the power generation, but they are not considered to be ideal cathode reactants due to toxicity, nonsustainability, and cost issues. ${ }^{[3]}$ Oxygen is a cost-effective, sustainable, nature-friendly, and scalable electron acceptor for practical applications. However, the slow rate and high overpotential of the oxygen reduction reaction (ORR) are the two critical issues ${ }^{[4]}$ that limit the performance of MFCs. There are two different chemical pathways (two- and four- electron) through which oxygen is reduced to hydrogen peroxide or water. The two-electron pathway exhibits a high overpotential for ORR, which is less efficient than the four-electron pathway normally observed with precious-metal based catalysts. ${ }^{[4,5]}$

Platinum-based catalysts have been regarded optimal for electrochemical reactions because they support the four-electron pathway and display low overpotential. However, the prohibitively high price of platinum prevents their use in large-scale applications. ${ }^{[6,7]}$ Thus, it is essential to develop low-cost ORR catalysts without sacrificing the performance of Pt-based catalysts. In the past several years, various catalysts have been proposed such as biocathodes (biofilm grown cathode), ${ }^{[8]}$ conductive polymers (polyaniline, ${ }^{[9]}$ polypyrrole ${ }^{[10]}$ ), macrocyclic compounds (Co-naphthalocyanine, ${ }^{[11]}$ pyrolyzed iron ethylenediaminetetraacetic ${ }^{[12,13]}$ ), metal oxides (manganese dioxide, ${ }^{[14,15]}$ lead oxide ${ }^{[16]}$ ), and carbon based (carbon nanotubes $(\mathrm{CNTs}),{ }^{[17]}$ graphene, ${ }^{[18,19]}$ activated carbon nanofibers ${ }^{[20]}$ ) materials. Recently, CNT-hybrid cathodes containing $\mathrm{Pt},{ }^{[21,22]} \mathrm{MnO}_{2},{ }^{[15,23,24]}$ chitosan, ${ }^{[25]}$ and iron phthalocyanine $(\mathrm{FePc})^{[26]}$ have displayed 
promising properties such as low cost and improved ORR activities mainly due to the large surface area and good conductivity of CNTs. Moreover, their high mechanical strength and excellent chemical stability have contributed to the development of durable cathodes. Nevertheless, power generation and stability with these catalysts are typically inferior to those of conventional Pt-based catalysts, or/and their synthesis processes are too complicated to be viable in practice.

Here we fabricated three-dimensional (3D) nitrogen-enriched iron-coordinated CNT (N/Fe-CNT) sponges as high-performance cathodes using simple and low-cost fabrication processes. A two-stage chemical vapor deposition (CVD) method was used to synthesize a selfassembled 3D framework consisting of intertwined nitrogen-enriched CNTs. The structure and chemical composition of the $\mathrm{N} / \mathrm{Fe}-\mathrm{CNT}$ sponge were investigated by scanning/transmission electron microscopy (SEM/TEM), X-ray diffraction (XRD), X-ray photoelectron spectroscopy (XPS), and Raman spectroscopy. The performances of the N/Fe-CNT-loaded MFCs were experimentally obtained using wastewater in anode chambers, and compared with that of MFCs with commercial Pt/C-coated carbon cloth (Pt-CC) cathodes. Cyclic voltammetry (CV) and rotating disk electrode (RDE) tests were also conducted to evaluate the electrocatalytic activity for ORR.

Pristine CNT sponges were formed at the end of the first stage of the synthesis process, and a nitrogen doping process was carried out at a higher temperature in the second stage (see the experimental section). The form of the as-synthesized N/Fe-CNT sponge (Figure 1a) reflects the cylindrical shape of a quartz furnace tube in which the sponge was synthesized, and no fracture was observed even with $90^{\circ}$-bending (Figure S1). The self-standing and deformable features differentiate the N/Fe-CNT sponge from other catalysts previously reported for MFC 
cathodes, ${ }^{[17,18,21,27-29]}$ which require an additional catalyst loading layer such as carbon cloth and carbon paper due to their powdery catalysts. The additional catalyst loading layer will not only add extra cost to MFCs but also increase the internal resistance of cathode.

SEM images in Figure $\mathbf{1 b}$ and c show that CNTs were randomly oriented and intertwined, creating porous 3D sponge-like frameworks. A post-treatment with pyridine at $800{ }^{\circ} \mathrm{C}$ generated small aggregates composed of carbon and iron compounds on the wall of CNTs (Figure 1c and d). In addition, a high-resolution TEM (HRTEM) image (Figure 1e) revealed that the inner walls of CNTs were composed of more ordered graphitic layers compared to the less ordered outer walls due to the nitrogen incorporation. The graphitic structure ensures a high electrical conductivity, which is important in charge transfer processes during ORR. ${ }^{[30]}$

In the XRD pattern of the N/Fe-CNT sponge (Figure 2a), the peak at $26.2^{\circ}$ corresponds to (002) planes of concentric graphitic carbon, which indicates the interlayer distance of $0.34 \mathrm{~nm}$ (close to $0.35 \mathrm{~nm}$ measured from the HRTEM image shown in Figure 1e) according to the Bragg's law. Other peaks were assigned to iron or iron-carbon compounds $\left(\mathrm{Fe}_{3} \mathrm{C}\right.$ and $\left.\mathrm{Fe}_{15.1} \mathrm{C}\right)$. No peak associated with nitrogen-containing compounds was found in the XRD result, suggesting nitrogen was mostly incorporated into the graphitic carbon (e.g., pyridinic and graphitic nitrogen) or amorphous iron-nitrogen compounds. Raman spectra (Figure 2b), therefore, were taken to investigate the effect of nitrogen incorporation into the graphitic carbon structure. The Raman spectra of both pristine CNT sponge and N/Fe-CNT sponge displayed Dband $\left(\sim 1330 \mathrm{~cm}^{-1}\right)$ and G-band peaks $\left(\sim 1572 \mathrm{~cm}^{-1}\right)$ of graphitic materials. ${ }^{[31]}$ The D-band is associated with a double resonance effect by defects, while the G-band is related to the in-plane vibration mode of $\mathrm{sp}^{2}$ carbon. ${ }^{[32]}$ The intensity ratio of the D-band to G-band $\left(\mathrm{I}_{\mathrm{D}} / \mathrm{I}_{\mathrm{G}}\right)$ qualitatively indicates the crystallinity of the graphitic carbon structure. $I_{D} / I_{G}$ is higher for $N / F e-C N T$ sponge 
(0.81) compared to CNT sponge (0.44), suggesting disordered structures induced by the nitrogen incorporation. ${ }^{[33]}$

The chemical structure and composition of the N/Fe-CNT sponge were further characterized by XPS (Figure S2), revealing the presence of carbon, nitrogen, oxygen, and iron, which is consistent with the elements in the precursors. The high-resolution scan of N 1s (Figure 2c) displayed two components corresponding to the pyridinic nitrogen (398.4 eV) and graphitic nitrogen $(401.0 \mathrm{eV})$. The pyridinic nitrogen is bonded with two carbon atoms, contributing one p-electron to the conjugated $\pi$ system, having a lone pair of electrons positioned in plane with the graphitic carbon. The graphitic nitrogen is bonded with three carbon atoms by replacing the original carbon atoms in the graphitic plane. Their structures are schematically shown in Figure 2d. These nitrogen groups were intentionally made with the pyridine precursor since they are essential in improving the catalytic activity of the carbon-based catalysts for ORR. ${ }^{[30]}$ The nitrogen composition was found to be $\sim 2 \%$ according to the XPS results (Table S1, Figure S2).

Four different N/Fe-CNT sponges (N/Fe-CNT10, N/Fe-CNT12, N/Fe-CNT15, N/FeCNT18) were tested as cathodes, as listed in Table 1, to investigate their performances for power generation in two-chamber MFCs (20-mL each for cathode and anode chambers). The variation in the tested samples was designed to identify the best pore volume per electrode mass (or surface area) as well as to investigate the dependency of power generation on electrode mass (or surface area). For ORR, the pore volume per mass is closely related to oxygen diffusion and the effective electrical conductivity, while the mass of the CNT sponge is proportional to its active surface area. Note that we used mass as an indicator of the electrode surface area, which is estimated to be $19 \mathrm{~m}^{2}$ per gram of $\mathrm{CNT} \cdot{ }^{[34]}$ For comparison, we used $\mathrm{Pt}-\mathrm{CC}$ whose single side was loaded with $\mathrm{Pt} / \mathrm{C}$ and then cut into two squares $\left(1.8 \times 1.8 \mathrm{~cm}^{2}\right)$. The two pieces were then 
attached using silver epoxy so that the Pt/C loaded sides were facing opposite each other and the $\mathrm{Pt} / \mathrm{C}$ were in direct contact with the solution during MFC testing. The thickness of the single PtCC sheet was measured to be $0.35 \mathrm{~mm}$. The area of the 2D Pt-CC $\left(3.2 \mathrm{~cm}^{2}\right)$ was chosen to be close to the cross sectional area of the 3D N/Fe-CNT samples $\left(3.1 \mathrm{~cm}^{2}\right)$. This design allowed us to investigate the influence of porous structures on MFC performance. The anode was made of $1 \times 1 \mathrm{~cm}^{2}$ carbon felt (same size for all testing), and all other conditions were kept the same.

Five MFCs loaded with N/Fe-CNT10, N/Fe-CNT12, N/Fe-CNT15, N/Fe-CNT18, and Pt$\mathrm{CC}$ cathodes were constructed and tested in a batch mode. Figure 3a shows the output voltage as a function of time over 3 months with a $1500-\Omega$ external resistor for N/Fe-CNT12 and Pt-CC cathodes. Both cells demonstrated negligible degradation over 3 months, and the N/Fe-CNT loaded cell showed slightly higher output voltage, indicating excellent stability and activity. We also obtained volumetric power densities based on anode chamber volume $(20 \mathrm{~mL})$ at steady power production stages (Figure 3b). The maximum power densities of N/Fe-CNT12 (14.1 W $\left.\mathrm{m}^{-3}\right)$ and Pt-CC $\left(12.8 \mathrm{~W} \mathrm{~m}^{-3}\right)$ loaded MFCs were similar, but N/Fe-CNT10 loaded MFC, which is shorter than N/Fe-CNT12, had a lower maximum power density $\left(9.1 \mathrm{~W} \mathrm{~m}^{-3}\right)$. The longer N/FeCNT15 and N/Fe-CNT18 resulted in higher maximum power densities compared to the MFCs with N/Fe-CNT12 and N/Fe-CNT10.

However, as for the MFCs with the two highest maximum power densities, the shorter N/Fe-CNT15 $\left(20.3 \mathrm{~W} \mathrm{~m}^{-3}\right)$ produced higher power than the longer N/Fe-CNT18 $\left(15.4 \mathrm{~W} \mathrm{~m}^{-3}\right)$. We believe that this trend in the power density is related to the competing effects caused by the oxygen diffusion/reduction rate, the effective electrical conductivity, and the active surface area, which are closely related to the pore volume per cathode mass and the cathode mass (or surface area). As shown in Figure 3c, the volumetric (anode volume) power produced per cathode mass 
increased with the enlarged pore volume per cathode mass until it reaches $11.4 \mathrm{~cm}^{3} / \mathrm{g}(\mathrm{N} / \mathrm{Fe}$ CNT15). Then, this power density dropped when the pore volume per cathode mass was further raised to $14.7 \mathrm{~cm}^{3} / \mathrm{g}(\mathrm{N} / \mathrm{Fe}-\mathrm{CNT} 18)$. The initial increase in the power per cathode mass, which indicates the productivity of cathode by normalization with cathode mass, suggests that the reaction is strongly influenced by oxygen supply. Smaller pore volumes may result in insufficient oxygen conditions (lack of electron acceptors) due to limited oxygen diffusion, thereby retarding ORR. Table 1 displays the average pore volumes calculated with CNTs whose outer/inner diameters and density were estimated to be $150 / 20 \mathrm{~nm}$ and $2.2 \mathrm{~g} \mathrm{~cm}^{-3}$, respectively. The power density drop for N/Fe-CNT18 (Figure 3c) is likely to come from the lower effective electrical conductivity compared to N/Fe-CNT15. When sponges are too porous, the number of electrically connected junctions between CNTs are small, resulting in less effective electron conduction through the CNTs. This may also suggest that the pore volume in N/Fe-CNT15 is large enough for sufficient oxygen supply. On the other hand, more active mass resulted in larger volumetric power density, as observed from the power density of N/Fe-CNT10, N/Fe-CNT18, and N/Fe-CNT15 (Figure 3d). Despite the larger cathode mass of N/Fe-CNT12 than that of $\mathrm{N} / \mathrm{Fe}-\mathrm{CNT} 18$, the power generation from N/Fe-CNT12 and N/Fe-CNT18 was similar, which could be attributed to the smaller pore volume in N/Fe-CNT12 (Figure 3c). Additionally, polarization curves of all cathodes showing voltage with respect to current density were compared in Figure S3.

In order to uncover the reason behind the high performance of the N/Fe-CNT sponges as cathodes for MFCs, electrochemical properties of the N/Fe-CNT sponges were further characterized by $\mathrm{CV}$ and $\mathrm{RDE}$ tests. In both tests, the catalysts (N/Fe-CNT sponge and $\mathrm{Pt} / \mathrm{C}$ ) were made into catalyst ink and then dropped on glassy carbon electrodes followed by drying 
(see the experimental part). According to the $\mathrm{CV}$ results (Figure 4a) in $\mathrm{O}_{2}$-saturated 50-mM phosphate buffer solution (PBS) $(\mathrm{pH}=7.6)$, strong oxygen reduction peaks were observed for both N/Fe-CNT and Pt/C. The resemblance of the peak location and intensity suggests similar catalytic activity for ORR.

RDE tests for both N/Fe-CNT (Figure 4b) and Pt/C (Figure S4a) were performed in the same $\mathrm{O}_{2}$-saturated $50 \mathrm{mM}$ PBS with rotating speed changing from $1600 \mathrm{rpm}$ to $850 \mathrm{rpm}$ at 150 rpm interval. The inclined plateaus from N/Fe-CNT near $-0.6 \mathrm{~V}$ vs $\mathrm{Ag} / \mathrm{AgCl}$, compared to reltaively flat one from $\mathrm{Pt} / \mathrm{C}$, is likely to be caused by the porous structure of our catalysts ${ }^{[35]}$ as well as nonuniformity of catalytic sites. ${ }^{[36]}$ Oxygen accessibility of some catalytic sites in the porous structure is presumably different from others due to tortuous paths and carbonaceous clumps having different accessbility, which results in lower potentials and/or longer diffusion time. ${ }^{[37-39]}$ On the other hand, according to XPS results, our samples contain different kinds of ORR active sites such as pyridinic nitrogen, graphitic nitrogen, and their coordination with iron. Since different catalytic sites may participate in ORR at different ranges of potentials, our multicatalytic sites would have resulted in an inclined plateau as a whole, which has been explained using a model with different electrocatalytic sites. ${ }^{[36]}$ The Koutecky-Levich plots were generated based on the RDE test results, as shown in Figure 4c and Figure S4b respectively for N/Fe-CNT and $\mathrm{Pt} / \mathrm{C}$. According to the Koutecky-Levich equation, $J^{-1}=J_{K}^{-1}+B^{-1} \omega^{-1 / 2}$ and $B=$ $0.62 n F C D^{2 / 3} v^{-1 / 6}$, where $J_{K}$ is the kinetic limiting current density, $\omega$ is the rotational speed, $n$ is the number of electron transferred, $F$ is the Faraday constant $\left(F=96485 \mathrm{C} \mathrm{mol}^{-1}\right), C$ is the bulk concentration of $\mathrm{O}_{2}\left(1.3 \times 10^{-6} \mathrm{~mol} \mathrm{~cm}\right), D$ is the diffusion coefficient of $\mathrm{O}_{2}\left(2.7 \times 10^{-5} \mathrm{~cm}^{2} \mathrm{~s}^{-}\right.$ $\left.{ }^{1}\right)$, and $v$ is the kinematic viscosity of the solution $\left(0.01 \mathrm{~cm}^{2} \mathrm{~s}^{-1}\right)$, there is a linear relation between $J^{1}$ and $\omega^{-1 / 2}$ and the slope depends on the number of electrons involved in the reaction. The 
linear relation and similar slopes for both $\mathrm{N} / \mathrm{Fe}-\mathrm{CNT}$ sponge and $\mathrm{Pt} / \mathrm{C}$ indicate the four-electron process, suggesting the efficient one-step ORR $\left(\mathrm{O}_{2}+4 \mathrm{H}^{+}+4 \mathrm{e}^{-} \rightarrow \mathrm{H}_{2} \mathrm{O}\right)$.

We also investigated two important aspects on the practicability of our MFCs, which are long-term stability and material/manufacutring cost of cathode. Stability is one of the most important aspects, particularly for non-precious metal based ORR catalysts. To investigate the stability of N/Fe-CNT sponge, it was cycled between $-0.5 \mathrm{~V}$ and $0.1 \mathrm{~V}$ for 20000 cycles followed by RDE test at $1000 \mathrm{rpm}$. The results are presented in Figure $4 \mathrm{~d}$ together with those from $\mathrm{Pt} / \mathrm{C}$ for comparison. After 20000 cycles, the degradation of N/Fe-CNT was smaller than that of Pt/C, but both N/Fe-CNT and Pt/C were marginally degraded with similar limiting current densities and voltage shifts.

The high price of MFCs is a major hurdle for their wide deployment, mainly due to the prohibitive cost of Pt catalysts. Powdery $\mathrm{Pt} / \mathrm{C}$ catalysts also require additional catalyst loading materials and processes, further increasing the manufacturing cost of MFC electrodes. Nonprecious metal based catalysts such as nitrogen-doped graphene, ${ }^{[28]}$ nitrogen-enriched $\mathrm{Fe} / \mathrm{Fe}_{3} \mathrm{C}$ carbon nanorods, ${ }^{[29]}$ and nitrogen-doped $\mathrm{CNTs}^{[17]}$ typically involve complicated and long fabrication steps. Although their initial power generation performances are comparable to those of their Pt/C electrodes, these systems may not be practically viable. Here we compared the raw material costs of our N/Fe-CNT sponge and Pt/C (not including catalyst loading layer), as shown in Table S2. It is striking that the cost of $1 \mathrm{~g} \mathrm{~N} / \mathrm{Fe}-\mathrm{CNT}$ sponge is only $\$ 0.69$, which is about $1.4 \%$ of $1 \mathrm{~g} \mathrm{Pt} / \mathrm{C}\left(\$ 50 \mathrm{~g}^{-1}\right)$. Furthermore, it is much cheaper than previously reported alternative catalysts with comparable performances as that of $\mathrm{Pt} / \mathrm{C},{ }^{[18,29]}$ to the best of our knowledge.

In summary, we have demonstrated a facile and low-cost method of synthesizing nitrogen/iron-coordinated CNTs in 3D sponge forms, which were directly used as self-standing 
cathodes for MFCs. The N/Fe coordination along with highly conductive porous CNTs resulted in high-power generation and long-term stability, which are comparable to those from Pt/Cbased MFCs, demonstrating its potential as a promising alternative to expensive $\mathrm{Pt} / \mathrm{C}$ loaded cathodes. We also found that the pore volume and cathode mass of the 3D sponges need to be optimized to maximize the performance, which may pave the way for future development of precious-metal-free self-standing cathodes for MFCs. The excellent MFC performances with the $\mathrm{N} / \mathrm{Fe}-\mathrm{CNT}$ sponge cathodes can be attributed to the efficient four-electron ORR process according to the Koutecky-Levich plots from the RDE tests as well as the high catalytic activity according to the $\mathrm{CV}$ results. The distinct features of our N/Fe-CNT sponges include self-standing characteristics suitable for cathode without conducting substrates (e.g., carbon cloth/paper) or additional steps (e.g., catalyst loading process), as well as considerably lower material/manufacturing costs with performances comparable to those of precious metal loaded MFCs. To the best of our knowledge, the synthesis cost of our N/Fe-CNT sponge is by far the lowest for MFC cathodes with performances similar to Pt/C loaded cathodes, which may bring MFCs and related fuel cell technologies one step closer to practical large-scale applications.

\section{Experimental Section}

Preparation of N/Fe-CNT sponges: Sponge-like porous 3D CNTs were first synthesized using a CVD method in a three-zone tube furnace equipped with a quartz tube (inner diameter: $\sim 22 \mathrm{~mm}$ ). Details can be found from our previous work. ${ }^{[30]}$ Without taking out the as-synthesized CNT sponge from the quartz tube, the furnace temperature was increased to $800{ }^{\circ} \mathrm{C}$. As soon as the reaction zone temperature reached $800{ }^{\circ} \mathrm{C}$, pyridine (Alfa Aesar, 99+\%) was injected into the tube using a syringe pump (Model KDS-100, KD Scientific) at a feeding rate of $20 \mathrm{~mL} \mathrm{~h}^{-1}$, while 
argon gas and hydrogen gas were flowed at $1000 \mathrm{sccm}$ and $100 \mathrm{sccm}$, respectively. The total amount of pyridine injected was proportional to the mass of pristine CNT sponge with a ratio of $25 \mathrm{~mL}$ of pyridine to $1 \mathrm{~g}$ of CNT sponge. After $1 \mathrm{~h}$ reaction, the quartz tube was naturally cooled under 200-sccm argon flow to room temperature.

Materials Characterization: The morphology and microstructure of the N/Fe-CNT sponge were characterized with SEM (FEI Quanta 600) and TEM (JEOL JEM-2010). The crystalline structure of the N/Fe-CNT sponge was studied by XRD measurement (Bruker-AXS D8 VARIO with $\mathrm{Cu}$ $\mathrm{K} \alpha$ X-ray) and XPS (Omicron XPS/UPS system with Argus detector, $\mathrm{Mg} \mathrm{K} \alpha$ as the X-ray source) was conducted to reveal the chemical composition and environment of the N/Fe-CNT sponge. A survey scan was first recorded with $1.0 \mathrm{eV}$ step size, followed by high-resolution scans of C 1s, N 1s, O 1s, and Fe 2p, using 0.05 eV step size. Raman spectroscopy was carried out on Horiba Jobin-Yvon LabRam with an excitation wavelength of $632.8 \mathrm{~nm}$ at room temperature.

MFC configuration and operation: The air bubbled two-chamber MFCs with a total volume of $40 \mathrm{~mL}$ ( $\sim 20 \mathrm{~mL}$ each for cathode and anode) were used to test the performance of all cathodes (N/Fe-CNT sponges and Pt-CC). Cylindrical acrylic chambers with $3 \mathrm{~cm}$ in inner diameter and height were fabricated using a milling machine (MDX-40, Roland DGA). Carbon felt $\left(1 \times 1 \mathrm{~cm}^{2}\right)$ (Morgan, UK) was used as anode in all MFCs. Titanium wires were permanently glued to the electrodes using silver epoxy for electrical connection. After placing proton exchange membranes (Nafion $117^{\mathrm{TM}}$, Ion Power Inc.) with silicone rubber gaskets, the chambers were screwed tightly to prevent leakage. The anode chambers were inoculated with 20 -vol $\%$ anaerobically activated sludge (Austin Wastewater Treatment Plant, Texas, USA) and 80-vol\% medium solution containing PBS (100 mM) and autoclaved anaerobic nutrient mineral buffer 
$(\mathrm{NMB}, \mathrm{pH} 7.0)^{[40,41]}\left(\mathrm{PBS}: \mathrm{NMB}=37: 1\right.$ by vol) with sodium acetate $\left(2 \mathrm{~g} \mathrm{~L}^{-1}\right)$. The cathode chambers were filled with $100 \mathrm{mM}$ PBS as catholyte and continuously purged with air to supply oxygen. When the voltage dropped below $\sim 50 \mathrm{mV}$, the anolyte was replaced with fresh one and then nitrogen gas was purged for 15 min to remove oxygen in the anode chambers. The anolyte was mixed using a magnetic stirrer $(350 \mathrm{rpm})$ during the experiment. Cell voltages across an external resistor $(1500 \Omega)$ were recorded every 2 min using a data acquisition system (National Instruments) via a LabView ${ }^{\mathrm{TM}}$ (National Instruments) interface. ${ }^{[42,43]}$ Polarization curves were obtained by varying the load resistor from $10 \mathrm{k} \Omega$ to $50 \Omega$ when the voltage was stable after several operation cycles. Power $(P)$ curves were calculated using $P=V \times I$ with the recorded voltage $(V)$ and measured current $(I)$ with the load resistors. The current and power densities were normalized by the volume $(20 \mathrm{~mL})$ of the anode chamber.

Electrochemical measurements: The electrochemical measurements were conducted on an electrochemical workstation (CHI 604D, CH Instrument) with a three-electrode system $(\mathrm{Ag} / \mathrm{AgCl}$ as a reference electrode, $\mathrm{Pt}$ wire as a counter electrode, and sample mounted glassy carbon electrode as a working electrode). To mount the samples on glassy carbon electrodes, catalyst ink was made by mixing 7-mg N/Fe-CNT sponge or Pt/C (20-wt\% Pt, Fuel Cell Earth), 160- $\mu \mathrm{L}$ 5-wt\% Nafion solution (Fuel Cell Earth), 170- $\mu \mathrm{L}$ ethanol (EMD Millipore, 92\%-94\%), and $670-\mu \mathrm{L}$ deionized water, followed by sonication for $30 \mathrm{~min}$. Then $5-\mu \mathrm{L}$ catalyst ink was drop-casted on the glassy carbon electrode resulting an areal loading of $0.5 \mathrm{mg} \mathrm{cm}^{-2}$. Both $\mathrm{CV}$ and $\mathrm{RDE}$ tests were performed in a $\mathrm{O}_{2}$-saturated (bubbling oxygen gas for $30 \mathrm{~min}$ before each test) 50-mM PBS ( $\mathrm{pH}=7.6)$ solution. $\mathrm{CV}$ tests were recorded in a potential range of $-1.0 \sim 0.5 \mathrm{~V}$ with a scan rate of $0.1 \mathrm{~V} \mathrm{~s}^{-1}$. RDE tests were conducted at different rotating speeds (1600 rpm to $850 \mathrm{rpm}$ with $150 \mathrm{rpm}$ interval) between $-0.7 \mathrm{~V}$ and $0.4 \mathrm{~V}$ at $0.01 \mathrm{~V} \mathrm{~s}^{-1}$ scan rate. Stability tests 
were performed by cycling between $-0.5 \mathrm{~V}$ and $0.1 \mathrm{~V}$ with a scan rate of $0.1 \mathrm{~V} \mathrm{~s}^{-1}$ for 20000 cycles followed by RDE test at $1000 \mathrm{rpm}$ with a scan rate of $0.01 \mathrm{~V} \mathrm{~s}^{-1}$.

\section{Acknowledgements}

This work was supported by the Qatar National Research Foundation (QNRF) grants, \#NPRP 5671-2-278 and \#NPRP 7-1634-2-604. 


\section{References}

[1] B. E. Logan, K. Rabaey, Science 2012, 337, 686-690.

[2] G. Reguera, K. D. McCarthy, T. Mehta, J. S. Nicoll, M. T. Tuominen, D. R. Lovley, Nature 2005, 435, 1098-1101.

[3] H. Rismani-Yazdi, S. M. Carver, A. D. Christy, O. H. Tuovinen, J. Power Sources 2008, 180, 683-694.

[4] B. Erable, D. Féron, A. Bergel, ChemSusChem 2012, 5, 975-987.

[5] Y. Qiao, S.-J. Bao, C. M. Li, Energy Environ. Sci. 2010, 3, 544-553.

[6] R. A. Rozendal, H. V. M. Hamelers, K. Rabaey, J. Keller, C. J. N. Buisman, Trends Biotechnol. 2008, 26, 450-459.

[7] K. Ben Liew, W. R. W. Daud, M. Ghasemi, J. X. Leong, S. Su Lim, M. Ismail, Int. J. Hydrogen Energy 2014, 39, 4870-4883.

[8] L. Jourdin, S. Freguia, B. C. Donose, J. Chen, G. G. Wallace, J. Keller, V. Flexer, J. Mater. Chem. A 2014, 2, 13093-13102.

[9] Y. Ren, D. Pan, X. Li, F. Fu, Y. Zhao, X. Wang, J. Chem. Technol. Biotechnol. 2013, 88, 1946-1950.

[10] M. Lu, L. Guo, S. Kharkwal, H. n. Wu, H. Y. Ng, S. F. Y. Li, J. Power Sources 2013, 221, 381-386.

[11] J. R. Kim, J.-Y. Kim, S.-B. Han, K.-W. Park, G. D. Saratale, S.-E. Oh, Bioresour. Technol. 2011, 102, 342-347.

[12] X. Xia, F. Zhang, X. Zhang, P. Liang, X. Huang, B. E. Logan, ACS Appl. Mater. Interfaces 2013, 5, 7862-7866.

[13] L. Wang, P. Liang, J. Zhang, X. Huang, Bioresour. Technol. 2011, 102, 5093-5097.

[14] S. Khilari, S. Pandit, M. M. Ghangrekar, D. Das, D. Pradhan, RSC Adv. 2013, 3, 79027911.

[15] Y. Zhang, Y. Hu, S. Li, J. Sun, B. Hou, J. Power Sources 2011, 196, 9284-9289.

[16] J. M. Morris, S. Jin, J. Wang, C. Zhu, M. A. Urynowicz, Electrochem. Commun. 2007, 9, 1730-1734.

[17] L. Feng, Y. Yan, Y. Chen, L. Wang, Energy Environ. Sci. 2011, 4, 1892-1899.

[18] L. Feng, Y. Chen, L. Chen, ACS Nano 2011, 5, 9611-9618.

[19] Y. Liu, H. Liu, C. Wang, S.-X. Hou, N. Yang, Environ. Sci. Technol. 2013, 47, 1388913895.

[20] X. Yang, W. Zou, Y. Su, Y. Zhu, H. Jiang, J. Shen, C. Li, J. Power Sources 2014, 266, 36-42.

[21] X. Xie, M. Pasta, L. Hu, Y. Yang, J. McDonough, J. Cha, C. S. Criddle, Y. Cui, Energy Environ. Sci. 2011, 4, 1293-1297.

[22] H. Wang, Z. Wu, A. Plaseied, P. Jenkins, L. Simpson, C. Engtrakul, Z. Ren, J. Power Sources 2011, 196, 7465-7469.

[23] Y. Chen, Z. Lv, J. Xu, D. Peng, Y. Liu, J. Chen, X. Sun, C. Feng, C. Wei, J. Power Sources 2012, 201, 136-141.

[24] K. B. Liew, W. R. Wan Daud, M. Ghasemi, K. S. Loh, M. Ismail, S. S. Lim, J. X. Leong, Int. J. Hydrogen Energy.

[25] X.-W. Liu, X.-F. Sun, Y.-X. Huang, G.-P. Sheng, S.-G. Wang, H.-Q. Yu, Energy Environ. Sci. 2011, 4, 1422-1427. 
[26] Y. Yuan, B. Zhao, Y. Jeon, S. Zhong, S. Zhou, S. Kim, Bioresour. Technol. 2011, 102, 5849-5854.

[27] Y. Ahn, I. Ivanov, T. C. Nagaiah, A. Bordoloi, B. E. Logan, J. Power Sources 2014, 269, 212-215.

[28] L. Y. Feng, L. Q. Yang, Z. J. Huang, J. Y. Luo, M. Li, D. B. Wang, Y. G. Chen, Sci RepUk 2013, 3 .

[29] Z. H. Wen, S. Q. Ci, F. Zhang, X. L. Feng, S. M. Cui, S. Mao, S. L. Luo, Z. He, J. H. Chen, Adv Mater 2012, 24, 1399-1404.

[30] G. Yang, W. Choi, X. Pu, C. Yu, Energy Environ. Sci. 2015.

[31] E. B. Barros, N. S. Demir, A. G. Souza Filho, J. Mendes Filho, A. Jorio, G. Dresselhaus, M. S. Dresselhaus, Phys. Rev. B 2005, 71.

[32] Z. H. Ni, H. M. Wang, Y. Ma, J. Kasim, Y. H. Wu, Z. X. Shen, ACS Nano 2008, 2 , 1033-1039.

[33] B. Guo, Q. Liu, E. Chen, H. Zhu, L. Fang, J. R. Gong, Nano Lett. 2010, 10, 4975-4980.

[34] A. Peigney, C. Laurent, E. Flahaut, R. R. Bacsa, A. Rousset, Carbon 2001, 39, 507-514.

[35] S. L. Gojkovic, S. Gupta, R. F. Savinell, J Electroanal Chem 1999, 462, 63-72.

[36] R. Z. Jiang, F. C. Anson, J Electroanal Chem 1991, 305, 171-184.

[37] G. Tamizhmani, J. P. Dodelet, D. Guay, G. Lalande, J Electrochem Soc 1994, 141, 41-45.

[38] G. Lalande, G. Faubert, R. Cote, D. Guay, J. P. Dodelet, L. T. Weng, P. Bertrand, J Power Sources 1996, 61, 227-237.

[39] G. Lalande, R. Cote, D. Guay, J. P. Dodelet, L. T. Weng, P. Bertrand, Electrochim Acta 1997, 42, 1379-1388.

[40] C. Erbay, S. Carreon-Bautista, E. Sanchez-Sinencio, A. Han, Environ. Sci. Technol. 2014, 48, 13992-13999.

[41] C. Erbay, X. Pu, W. Choi, M.-J. Choi, Y. Ryu, H. Hou, F. Lin, P. de Figueiredo, C. Yu, A. Han, J. Power Sources 2015, 280, 347-354.

[42] H. Hou, L. Li, C. U. Ceylan, A. Haynes, J. Cope, H. H. Wilkinson, C. Erbay, P. d. Figueiredo, A. Han, Lab Chip 2012, 12, 4151-4159.

[43] H. Hou, L. Li, Y. Cho, P. de Figueiredo, A. Han, PloS one 2009, 4, e6570. 
Table 1 Characteristics of the tested cylindrical N/Fe-CNT sponge samples whose diameter is 20 $\mathrm{mm}$. The pore volumes are average values calculated with CNTs whose outer/inner diameters and density are respectively $150 / 20 \mathrm{~nm}$ and $2.2 \mathrm{~g} \mathrm{~cm}^{-3}$.

\begin{tabular}{lccccc}
\hline & $\begin{array}{c}\text { Length } \\
(\mathbf{m m})\end{array}$ & $\begin{array}{c}\text { Mass } \\
(\mathbf{m g})\end{array}$ & $\begin{array}{c}\text { Volume/Mass } \\
\left(\mathbf{c m}^{\mathbf{3}} \mathbf{g}^{-\mathbf{1}}\right)\end{array}$ & $\begin{array}{c}\text { Density } \\
\left(\mathbf{m g ~ c m}^{-3}\right)\end{array}$ & $\begin{array}{c}\text { Pore volume } \\
\left(\boldsymbol{\mu ~ m}^{\mathbf{3}}\right)\end{array}$ \\
\hline N/Fe-CNT10 & 10 & 362 & 8.67 & 115 & 0.796 \\
\hline N/Fe-CNT12 & 12 & 402 & 9.37 & 107 & 0.894 \\
\hline N/Fe-CNT15 & 15 & 413 & 11.4 & 87.7 & 1.239 \\
\hline N/Fe-CNT18 & 18 & 385 & 14.7 & 68.1 & 1.864 \\
\hline
\end{tabular}



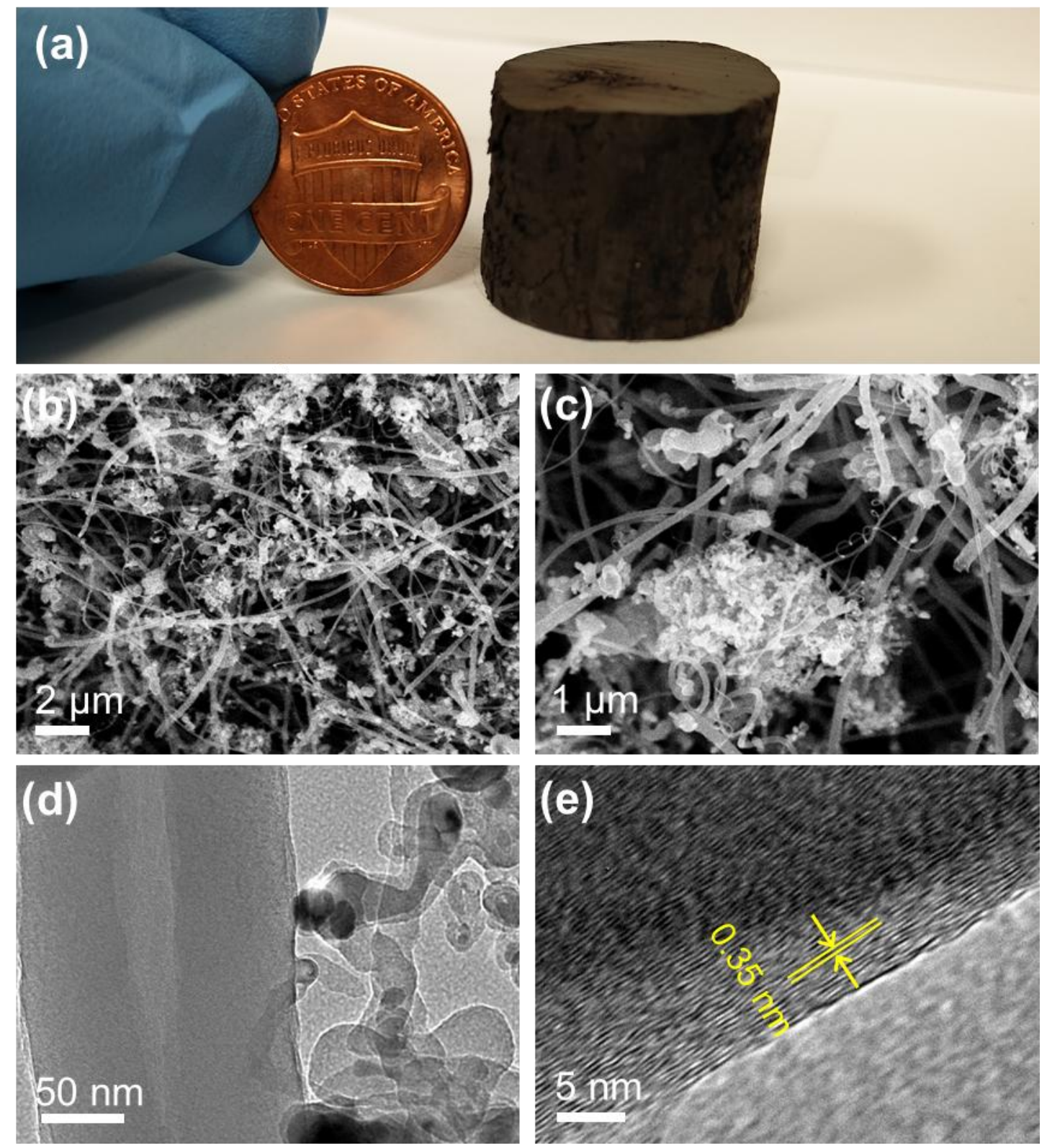

Figure 1. Morphology and structure of N/Fe-CNT. (a) An as-synthesized self-standing N/FeCNT sponge. (b-c) SEM images of N/Fe-CNT sponge showing small intertwined CNTs and small aggregates attached to the wall of CNTs. (d-e) TEM images of N/Fe-CNT showing graphitic layers of multi-wall CNTs. 

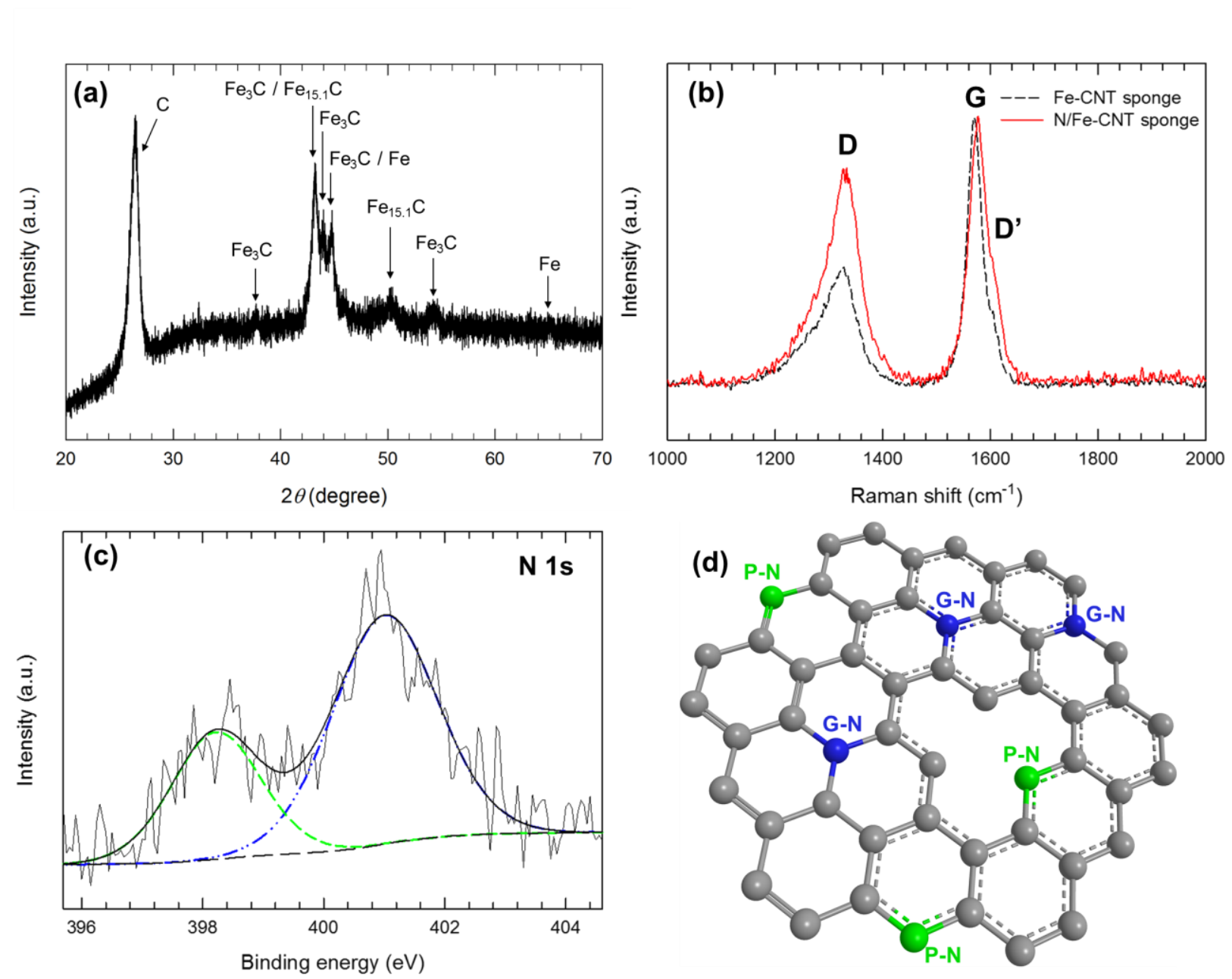

Figure 2. Crystal structure and chemical composition of N/Fe-CNT. (a) XRD pattern of a N/FeCNT sponge where carbon, iron, and iron compound peaks were identified. (b) Raman spectra of a pristine CNT sponge and a N/Fe-CNT sponge, indicating the increased number of defects after nitrogen incorporation. (c) High-resolution scan of $\mathrm{N} \mathrm{1s}$, deconvoluted into pyridinic nitrogen $(398.4 \mathrm{eV})$ and graphitic nitrogen $(401.0 \mathrm{eV})$. (d) Schematic representation of nitrogen incorporated graphitic structure. The spheres represent carbon and nitrogen atoms, and P-N and G-N respectively denote pyridinic nitrogen and graphitic nitrogen. 

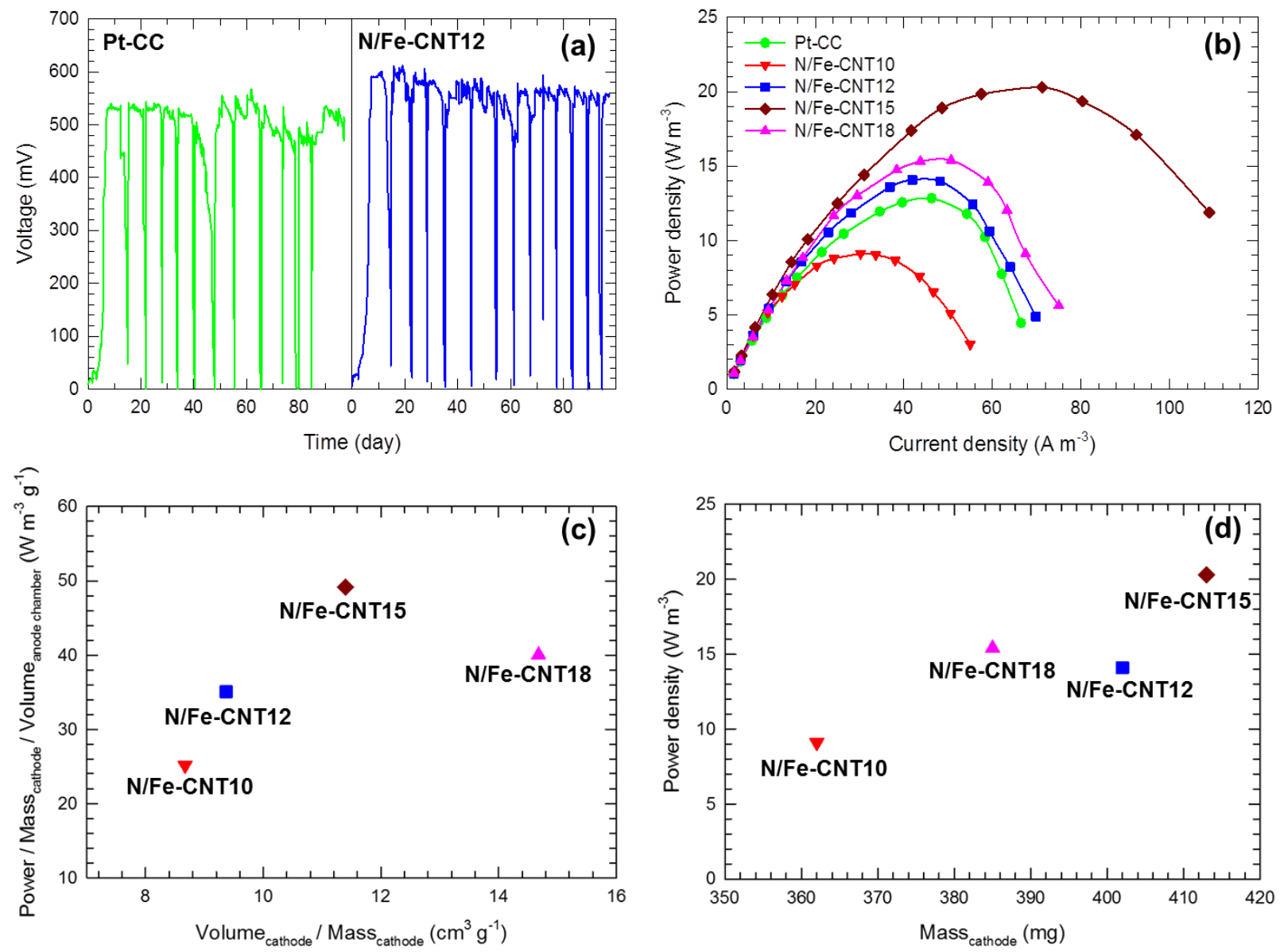

Figure 3. MFC performances with $\mathrm{N} / \mathrm{Fe}-\mathrm{CNT}$ sponge and Pt-CC cathodes. (a) Voltage output over $1.5 \mathrm{k} \Omega$ resistor from MFCs equipped with $\mathrm{N} / \mathrm{Fe}-\mathrm{CNT} 12$ and Pt-CC cathodes. (b) Polarization curves with all N/Fe-CNT sponges and Pt-CC cathodes. (c) Volumetric (anode volume) power produced per cathode mass with the change of pore volume per cathode mass. (d) Volumetric (anode volume) power with respect to the mass of cathodes. To obtain the volumetric power, the measured power was divided by the volume of the anode chamber $(20 \mathrm{~mL})$. 

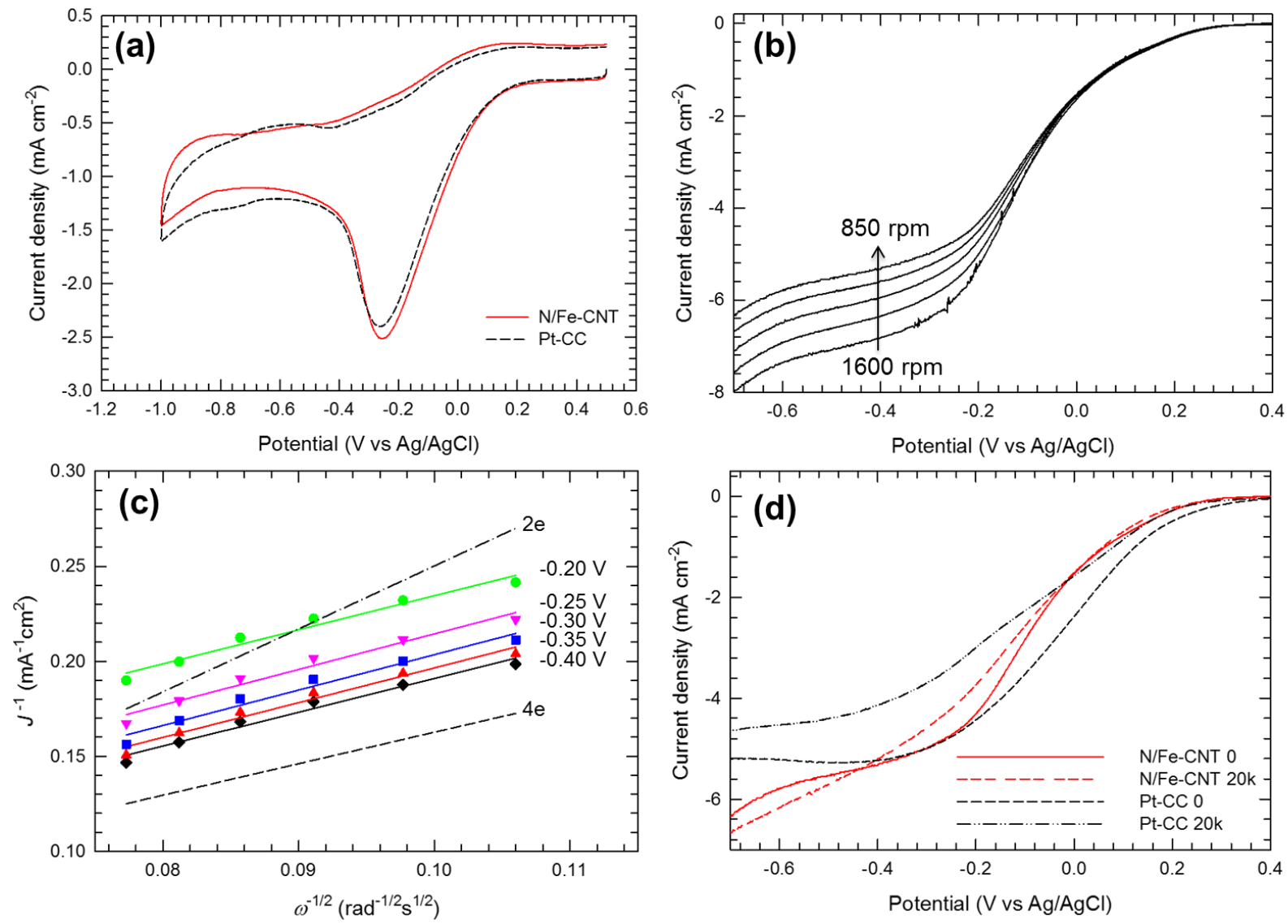

Figure 4. Electrochemical properties of N/Fe-CNT. (a) CV results of N/Fe-CNT and Pt/C catalysts showing strong oxygen reduction peaks. (b) $\mathrm{RDE}$ test results of $\mathrm{N} / \mathrm{Fe}-\mathrm{CNT}$ in $\mathrm{O}_{2}$ saturated $50 \mathrm{mM}$ PBS. The rotating speed was changed from $1600 \mathrm{rpm}$ to $850 \mathrm{rpm}$ with $150 \mathrm{rpm}$ interval. All data were recorded from $0.4 \mathrm{~V}$ to $-0.7 \mathrm{~V}$ with a scan rate of $0.01 \mathrm{~V} \mathrm{~s}^{-1}$. (c) Koutecky-Levich plots of N/Fe-CNT, indicating the 4-electron ORR process. (d) RDE test results $(1000 \mathrm{rpm})$ of N/Fe-CNT and Pt/C before and after $20000 \mathrm{CV}$ cycles. All electrochemical tests were performed in $\mathrm{O}_{2}$-saturated $50 \mathrm{mM}$ PBS. The scan rate was $0.1 \mathrm{~V} \mathrm{~s}^{-1}$ and $0.01 \mathrm{~V} \mathrm{~s}^{-1}$ for $\mathrm{CV}$ and RDE, respectively. 


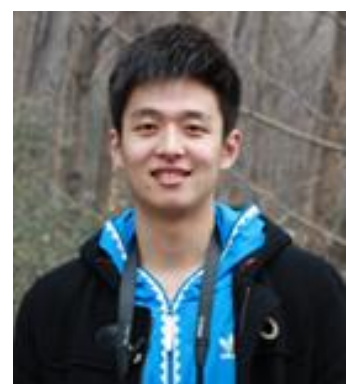

Gang Yang is currently a Ph.D. candidate in Professor Yu's research lab at Texas A\&M University (College station, Texas, USA). He received his B.S. degree in Chemistry from Nanjing University (China), and M.S. degree in Materials Science and Engineering from University of Dayton (United States). His research centers on designing three-dimensional nano-materials as electrodes and/or catalysts for lithium batteries, proton exchange membrane fuel cells, and microbial fuel cells.

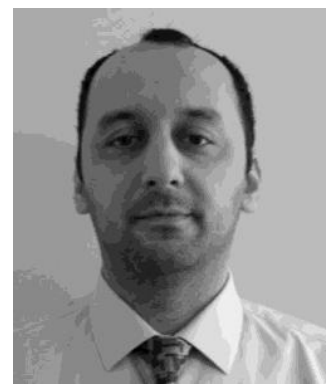

Celal Erbay received his B.S. degree in computer technology and information systems in 2006 from Bilkent University, Turkey, and the Master degree in computer engineering from Texas A\&M University in 2011. Currently, he is a Ph. D. candidate under the supervision of Prof. Arum Han at Electrical and Computer Engineering at the same university. His doctoral research focuses on the micro devices and energy harvesting technologies for bioenergy production from organic wastes and development of microbial electrochemical cell systems for wastewater treatment.

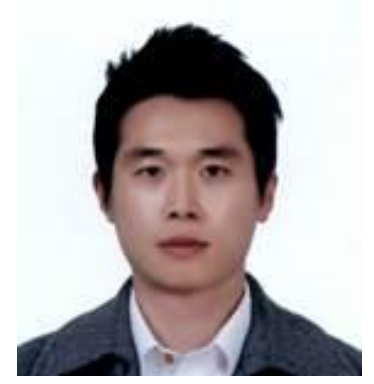

Su-in Yi is currently a Ph.D. candidate in Professor Yu's research lab at Texas A\&M University (College Station, Texas, USA). He received his B.S. degree in Electrical Engineering from SungKyunKwan University (S. Korea) and M.S. degree in Graduate School of EEWS (Energy, Environment, Water, and Sustainability) from Korea Advanced Institute of Science and Technology (S. Korea). His research interests include theoretical and experimental study of thermoelectric transport behaviors of nanostructured oxide and silicide materials. 


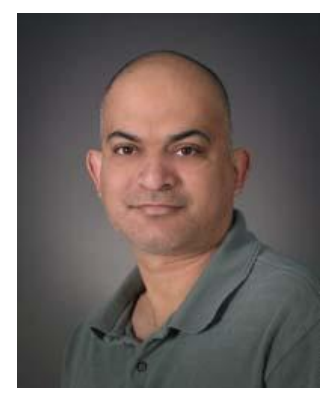

Paul de Figueiredo is an Associate Professor in the Department of Microbial Pathogenesis and Immunology at the Texas A\&M Health Center and a member of the Norman Borlaug Center at Texas A\&M University (College Station, TX). He obtained his Ph.D. degree from the Department of Biochemistry, Molecular and Cell Biology at Cornell University (1997), and then held postdoctoral research positions at MIT and the University of Washington School of Medicine prior to starting his position at Texas A\&M in 2005. He has broad expertise in microbiology, cell biology, and host-pathogen interactions, and is pursuing interdisciplinary research in clean energy technologies.

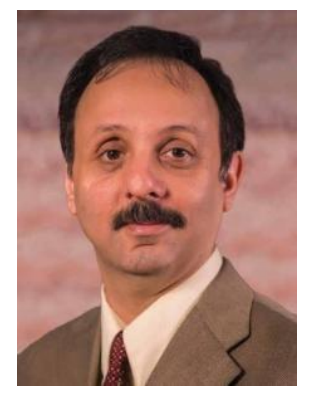

Reza Sadr is associate professor in the mechanical engineering program at Texas A\&M University Qatar. He received his $\mathrm{PhD}$ from University of Utah in 2002. Before joining 2008, he moved to Georgia Tech Atlanta as research scientist, and then to Georgia Tech Savannah as visiting assistant professor. His research expertise is in experimental methods in thermo-fluid sciences. His current research addresses microfluidics, droplets and sprays, environmental fluid mechanics, and supercritical thermo-fluids. He is a member of American Society of Mechanical Engineering and American Physical Society since 1998.

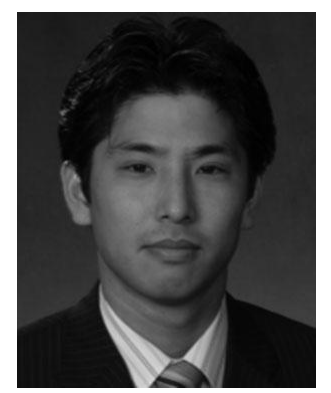

Arum Han received the B.S. degree from Seoul National University in 1997; the M.S. degree from the University of Cincinnati in 2000; and the Ph.D. degree from the Georgia Institute of Technology in 2005, all in electrical engineering. In 2005, he joined the Department of Electrical and Computer Engineering, Texas A\&M University as an Assistant Professor, and holding a Joint Faculty position in the Biomedical Engineering Department since 2006. He became an Associate Professor in 2011. His research interests include solving grand challenge problems in the broad area of health and energy through the use of micro/nano systems technology. 


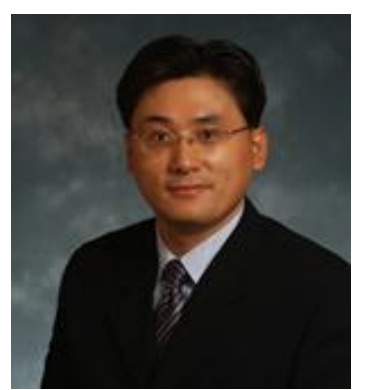

Choongho $\mathbf{Y u}$ is an associate professor of Mechanical Engineering and Materials Science and Engineering at Texas A\&M University (College station, Texas, USA). He obtained his Ph.D. degree from the Department of Mechanical Engineering at the University of Texas at Austin, USA (2004), and then joined Materials Sciences Division at Lawrence Berkeley National Laboratory, USA prior to starting his position at Texas A\&M University in 2007. His research centers on electronic and phononic transport behaviors in nanomaterials including batteries, thermoelectrics, and fuel cells. 